\title{
Glibenclamide, metformin, and insulin for the treatment of gestational diabetes: a systematic review and meta-analysis
}

\author{
(c) (1) (8) OPEN ACCESS
}

\author{
Montserrat Balsells registrar in endocrinology and nutrition ${ }^{1}$, Apolonia García-Patterson registrar \\ in endocrinology and nutrition ${ }^{2}$, Ivan Solà associate researcher ${ }^{345}$, Marta Roqué associate \\ researcher ${ }^{345}$, Ignasi Gich associate researcher ${ }^{567}$, Rosa Corcoy assistant professor in endocrinology \\ and nutrition ${ }^{289}$
}

${ }^{1}$ Department of Endocrinology and Nutrition, Hospital Universitari Mútua de Terrassa, Terrassa 8821, Spain; ${ }^{2}$ Department of Endocrinology and Nutrition, Hospital de la Santa Creu i Sant Pau, Barcelona 08025, Spain; ${ }^{3} \mid$ beroamerican Cochrane Centre, Hospital de la Santa Creu i Sant Pau, Barcelona; ${ }^{4}$ Institute of Biomedical Research (IIB Sant Pau), Hospital de la Santa Creu i Sant Pau Barcelona; ${ }^{5} \mathrm{CIBER}$ Epidemiología y Salud Pública (CIBERESP), Instituto de Salud Carlos III, Madrid 28029, Spain; ${ }^{6}$ Department of Epidemiology, Hospital de la Santa Creu i Sant Pau, Barcelona; ${ }^{7}$ Department of Clinical Pharmacology and Therapeutics, Universitat Autònoma de Barcelona, Bellaterra 08193 (Cerdanyola del Vallès), Spain; ${ }^{8}$ CIBER Bioengineering, Biomaterials and Nanotechnology (CIBER-BBN), Instituto de Salud Carlos III, Madrid; ${ }^{9}$ Department of Medicine, Universitat Autònoma de Barcelona, Bellaterra

\begin{abstract}
Objective To summarize short term outcomes in randomized controlled trials comparing glibenclamide or metformin versus insulin or versus each other in women with gestational diabetes requiring drug treatment.

Design Systematic review and meta-analysis.

Eligibility criteria for selecting studies Randomized controlled trials that fulfilled all the following: (1) published as full text; (2) addressed women with gestational diabetes requiring drug treatment; (3) compared glibenclamide $v$ insulin, metformin $v$ insulin, or metformin $v$ glibenclamide; and (4) provided information on maternal or fetal outcomes.
\end{abstract}

Data sources Medline, CENTRAL, and Embase were searched up to 20 May 2014

Outcomes measures We considered 14 primary outcomes ( 6 maternal, 8 fetal) and 16 secondary ( 5 maternal, 11 fetal) outcomes.

Results We analyzed 15 articles, including 2509 subjects. Significant differences for primary outcomes in glibenclamide $v$ insulin were obtained in birth weight (mean difference $109 \mathrm{~g}$ (95\% confidence interval 35.9 to 181)), macrosomia (risk ratio 2.62 (1.35 to 5.08)), and neonatal hypoglycaemia (risk ratio 2.04 (1.30 to 3.20$)$ ). In metformin $v$ insulin, significance was reached for maternal weight gain (mean difference $-1.14 \mathrm{~kg}(-2.22$ to -0.06$)$ ), gestational age at delivery (mean difference -0.16 weeks $(-0.30$ to -0.02$)$ ), and preterm birth (risk ratio 1.50 (1.04 to 2.16)), with a trend for neonatal hypoglycaemia (risk ratio $0.78(0.60$ to 1.01)). In metformin $v$ glibenclamide, significance was reached for maternal weight gain (mean difference $-2.06 \mathrm{~kg}(-3.98$ to -0.14$)$ ), birth weight (mean difference $-209 \mathrm{~g}(-314$ to -104$)$ ), macrosomia (risk ratio $0.33(0.13$ to 0.81$)$ ), and large for gestational age newborn (risk ratio 0.44 (0.21 to 0.92$)$ ). Four secondary outcomes were better for metformin in metformin $v$ insulin, and one was worse for metformin in metformin $v$ glibenclamide. Treatment failure was higher with metformin than with glibenclamide.

Conclusions At short term, in women with gestational diabetes requiring drug treatment, glibenclamide is clearly inferior to both insulin and metformin, while metformin (plus insulin when required) performs slightly better than insulin. According to these results, glibenclamide should not be used for the treatment of women with gestational diabetes if insulin or metformin is available.

Systematic review registration NCT01998113

\section{Introduction}

Gestational diabetes is a common complication of pregnancy, and prevalence is increasing. ${ }^{1}$ The standard therapy for women with gestational diabetes requiring drug treatment is insulin. However, since the seminal trial by Langer et al comparing glibenclamide (glyburide) with insulin, ${ }^{2}$ oral agents have been 
increasingly viewed as potential alternatives. Several observational and randomized controlled trials have addressed the use of oral agents in gestational diabetes, mainly glibenclamide and metformin. ${ }^{3-6}$ Although neither glibenclamide nor metformin have marketing authorization for their use in pregnancy, their use as an adjunct therapy in gestational diabetes has been considered by several guidelines. For example, glibenclamide has been acknowledged in the Fifth International Workshop-Conference in Gestational Diabetes Mellitus, ${ }^{7}$ and both glibenclamide and metformin are considered in National Institute for Health and Care Excellence (NICE) guidance ${ }^{8}$ and American College of Obstetricians and Gynecologists (ACOG) practice bulletin. ${ }^{9}$ Oral agents are an attractive alternative to insulin for their easier administration, lower cost, and better acceptance..$^{10}$ Use of oral agents is increasing, and in some settings they are the first option when drug treatment is required for women with gestational diabetes. ${ }^{611-13}$ In a large nationwide retrospective cohort study in the US including 10778 women with drug treated gestational diabetes, use of glibenclamide increased from $7.4 \%$ in 2000 to $64.5 \%$ in 2011 , becoming the most common treatment since 2007. ${ }^{12}$

In recent years, several systematic reviews and meta-analyses have summarized the published evidence using different approaches: combining randomized controlled trials and observational studies addressing glibenclamide, ${ }^{14}$ performing a meta-analysis of randomized controlled trials comparing glibenclamide with insulin, ${ }^{15}$ combining randomized controlled trials addressing glibenclamide or metformin versus insulin in a single meta-analysis, ${ }^{16}$ and conducting a meta-analysis of randomized controlled trials comparing metformin with insulin. ${ }^{17}$ In 2013, the meta-analysis by Gui and colleagues, which included five randomized controlled trials comparing metformin with insulin, showed differences favouring metformin in terms of maternal weight gain and pregnancy induced hypertension but favouring insulin in terms of gestational age at delivery and preterm birth. ${ }^{17} \mathrm{~A}$ later meta-analysis addressing metformin versus insulin added a non-randomized controlled trial study. ${ }^{18}$ In 2014, the meta-analysis of Zeng and colleagues, including five randomized controlled trials comparing glibenclamide with insulin, showed differences favouring glibenclamide in terms of maternal hypoglycaemia but favouring insulin in terms of birth weight, macrosomia, and neonatal hypoglycaemia. ${ }^{15}$ As yet, randomized controlled trials comparing glibenclamide or metformin with insulin, or metformin with glibenclamide, have not been addressed in a comprehensive systematic review that evaluates the most relevant maternal and fetal outcomes.

The aim of the present study was to compare risks and benefits of insulin, glibenclamide, and metformin in terms of short term outcomes when used for women with gestational diabetes requiring drug treatment. By means of a systematic review, we synthesized the available evidence concerning short term maternal and fetal outcomes in randomized controlled trials that compared glibenclamide with insulin, metformin with insulin, or metformin with glibenclamide in women with gestational diabetes requiring drug treatment.

\section{Methods}

\section{Literature search and study selection}

The protocol of this systematic review was registered in ClinicalTrials.gov (NCT01998113), and endorses PRISMA. A comprehensive electronic search strategy was conducted in Medline, Cochrane Central Register of Controlled Trials, and Embase (see supplementary table 1). The last search update was performed on 20 May 2014. Bibliographies from relevant studies were reviewed, and authors of potentially eligible studies were contacted when necessary to request further information regarding study design or primary outcomes.

Studies were eligible if they fulfilled all the following: (1) they were randomized controlled trials; (2) subjects were women with gestational diabetes requiring drug treatment; (3) they compared glibenclamide with insulin, metformin with insulin, or metformin with glibenclamide; (4) they provided information on one or more maternal or fetal outcome; and (5) they were published as a full paper. The exclusion criterion was a significant overlap with other articles by the same group.

\section{Data collection and outcomes}

We pre-specified maternal and fetal outcomes of interest. Primary outcomes were defined as those considered relevant or commonly reported. Primary maternal outcomes were $\mathrm{HbA}_{1 \mathrm{c}}$ level in the third trimester, severe maternal hypoglycaemia, pre-eclampsia, total weight gain during pregnancy, caesarean section, and (for trials comparing metformin $v$ glibenclamide) treatment failure. Primary fetal outcomes were gestational age at delivery, preterm birth, birth weight, macrosomia ( $\geq 4000 \mathrm{~g}$ ), large for gestational age newborn (birth weight $>90$ th centile), small for gestational age newborn (birth weight $<10$ th centile), any neonatal hypoglycaemia, and perinatal mortality.

Secondary maternal outcomes were fasting and postprandial blood glucose, weight gain since study entry, pregnancy induced hypertension, and induction of labour. Secondary fetal outcomes were fetal hyperinsulinism (measured as either cord $\mathrm{C}$ peptide or cord insulin), abnormal Apgar score at 1 and 5 minutes, obstetric trauma, severe neonatal hypoglycaemia (as defined by authors, or requiring intravenous glucose or admission to the neonatal intensive care unit), neonatal jaundice, significant respiratory distress (described as respiratory distress syndrome or requiring respiratory support), stillbirth, neonatal mortality, and admission to the neonatal intensive care unit.

Two investigators independently screened abstracts, reviewed the full text, decided on eligibility, and collected data using a previously designed form. Discrepancies were solved after reviewing the source and by consensus with a third investigator. The risk of bias was assessed at the study level taking into account the randomization process, quality of allocation concealment, blinding, selective outcome reporting, sample size calculation, attrition rates, intention-to-treat analysis, and comparability of subjects' baseline characteristics (maternal age, pre-pregnancy body mass index, fasting plasma glucose concentration and two hours after oral glucose tolerance test, gestational age, and $\mathrm{HbA}_{1 \mathrm{c}}$ level at study entry). ${ }^{19}$ This assessment was essentially qualitative, except in the case of attrition rates where we predefined a quantitative criterion: a cut-off of $15 \%$ both for overall attrition rate and for differences between treatment groups (unless otherwise foreseen by the authors). This information concerning risk of bias was used to perform sensitivity analyses, excluding articles with relevant weaknesses in trial design or execution. Unbalanced baseline characteristics alone were not considered an exclusion criterion for the sensitivity analysis.

\section{Statistical analysis}

Outcome data from original studies were pooled into either relative risks for dichotomous outcomes or mean differences for continuous outcomes using Review Manager 5.1 software. Heterogeneity was explored for all the meta-analyses. Analyses were undertaken using a fixed effects model; in case of substantial heterogeneity $\left(\mathrm{I}^{2} \geq 50 \%\right)$ a random effects model was 
used. Pooled analyses were also used to compare baseline characteristics. In the metformin versus glibenclamide comparison including two studies, we performed a qualitative assessment of the effects (direction, magnitude, and precision) in addition to the meta-analysis.

In the metformin versus insulin comparison, the only baseline characteristic that presented significant and relevant differences between treatment groups was body mass index. Thus, for this comparison we performed a meta-regression to explore baseline body mass index as a source of heterogeneity by means of meta-regression models. Post hoc meta-regression models were built for variables known to be influenced by maternal body mass index and with heterogeneity $>10 \%$ (weight gain, caesarean section, macrosomia, and a large for gestational age newborn), considering the average baseline difference in body mass index between treatments as the independent factor.

In the three drug comparisons, we calculated average treatment failure $(\%)$ for oral agents. In the metformin versus glibenclamide comparison, we also calculated the pooled estimate for treatment failure in terms of risk ratio.

Publication bias was assessed for all analyses after visual inspection of funnel plots.

\section{Results}

The search retrieved 974 abstracts. As depicted in the flow chart (supplementary figure 1), 15 studies fulfilled our inclusion criteria—seven comparing glibenclamide with insulin (798 subjects), six comparing metformin with insulin (1362 subjects), and two comparing metformin with glibenclamide (349 subjects). The characteristics of the included studies and quality assessment are described in supplementary tables 2 and 3. Meta-analysis results for each comparison are presented in tables $1-3 \Downarrow \Downarrow \Downarrow$. Forest plots for primary outcomes with significant differences in any of the meta-analyses are shown as supplementary figure 2, with the exception of those for birth weight and any neonatal hypoglycaemia, which are displayed in figures $1 \Downarrow$ and $2 \Downarrow$.

\section{Glibenclamide versus insulin}

Characteristics of the seven included studies and baseline data are summarized in supplementary table $2 .^{2}{ }^{320-24}$ Studies were performed in the US, Brazil, and India. Most common diagnostic criteria were those of Carpenter and Coustan and of the World Health Organization, and blood glucose cut-off values for initiating drug treatment were similar among trials. The largest study ${ }^{2}$ included 404 subjects, while each of the other six included fewer than 100 participants. Baseline characteristics did not differ between groups (see supplementary table 4).

All trials were open label, and none had a published protocol. Randomization procedure, allocation concealment, and sample size calculation were not available for any of the studies. Three studies reported one variable less than indicated in the methods. ${ }^{20}{ }^{24}$ Two studies had attrition issues: one had a high balanced attrition, but, as this had been foreseen by the authors, ${ }^{3}$ the study was not excluded in the sensitivity analysis; the other study had an unbalanced attrition rate $(3 / 13(23.1 \%) v 0 / 13)$ (see supplementary table 3$)^{21}$ and was excluded in the sensitivity analysis.

When compared with insulin, glibenclamide was associated with a higher birth weight (pooled mean difference $109 \mathrm{~g}(95 \%$ confidence interval 35.9 to 181) and more macrosomia (pooled risk ratio 2.62 (1.35 to 5.08)) and neonatal hypoglycaemia (pooled risk ratio 2.04 (1.30 to 3.20$)$ ). The average treatment failure in the glibenclamide group was $6.37 \%$ (20/314). None of the secondary outcomes showed significant differences (table $1 \Downarrow$ ). The sensitivity analysis did not affect treatment estimates in any substantial way (see supplementary table 5), and funnel plots displayed symmetrical distributions for all the outcomes with the exception of large for gestational age.

Outcome definition and active ascertainment were not always provided. In some outcomes such as preterm birth, caesarean section, birth weight, and Apgar score there is wide agreement in their definition. In those where definition and detection protocol can display more differences (neonatal hypoglycaemia, respiratory distress, jaundice, and admission to the neonatal intensive care unit), reported definitions and active ascertainment were reasonably similar with the exception of the protocol to detect neonatal hypoglycaemia with measurements of glycaemia ranging from two to nine.

In addition to primary and secondary outcomes, maternal biochemical hypoglycaemia was reported to be higher in the insulin group by Langer et $\mathrm{al}^{2}{ }^{2}$ whereas Ogunyemi et $\mathrm{al}^{22}$ reported a similar rate in both groups. Ogunyemi et al also described additional side effects in $6.3 \%$ of patients treated with glibenclamide.

\section{Metformin versus insulin}

Characteristics of the six included studies and baseline data are summarized in supplementary table $2 .^{425-29}$ Studies were performed in the US, Australia and New Zealand, Finland, Iran, and Brazil. Several diagnostic criteria were used, and blood glucose cut-off values for initiating drug treatment were similar among studies. Metformin and insulin groups were similar in all baseline characteristics, but prepregnancy body mass index was significantly higher in the metformin group (pooled mean difference 0.78 (95\% confidence interval 0.15 to1.41), see supplementary table 4$)$.

All trials were open label, and sample size calculation was described. Published protocol, randomization procedure, and allocation concealment were available for most studies. All the studies reported findings for the outcomes specified in their protocols. Attritions were lower than $15 \%$ and balanced among groups in all trials. One study was excluded from the sensitivity analysis ${ }^{25}$ because of a relevant weakness in trial execution (when we calculated mean height using the reported weight and body mass index in the metformin and insulin groups, we obtained figures of 140 and $160 \mathrm{~cm}$ respectively, which is virtually impossible) (see supplementary table 3).

When compared with insulin, metformin was associated with less maternal weight gain (pooled mean difference $-1.14 \mathrm{~kg}$ (95\% confidence interval -2.22 to -0.06$)$ ), lower gestational age at delivery (pooled mean difference -0.16 weeks $(-0.30$ to $-0.02)$ ), and more preterm birth (pooled risk ratio 1.50 (1.04 to 2.16)). A trend was observed towards a lower rate of any neonatal hypoglycaemia (pooled risk ratio 0.78 (0.60 to 1.01$)$ ). The average treatment failure in the metformin group was $33.8 \%$ (229/678). For secondary outcomes, metformin was associated with lower postprandial blood glucose (pooled mean difference $-0.14 \mathrm{mmol} / \mathrm{L}(-0.22$ to -0.05$)$ ), less maternal weight gain since study entry (pooled mean difference $-1.23 \mathrm{~kg}(-1.72$ to $-0.73)$ ), less pregnancy induced hypertension (pooled risk ratio $0.53(0.31$ to 0.90$)$ ), and less severe neonatal hypoglycaemia (pooled risk ratio $0.62(0.42$ to 0.94$)$ ) (table $2 \Downarrow$ ). The sensitivity analysis did not affect treatment estimates in any substantial way (see supplementary table 6), and funnel plots displayed symmetrical distributions for all the outcomes, with the exception of maternal weight gain and weight gain since study 
entry. Outcome definition and active ascertainment were not always provided. In outcomes where definition and detection protocol can display more differences, reported definitions and active ascertainment were reasonably similar, with the exception of the protocol to detect neonatal hypoglycaemia with measurements of glycaemia ranging from one to more than six.

Baseline maternal body mass index continued to be higher in the metformin group in the sensitivity analysis (pooled mean difference 0.68 (0.05 to1.32)). Baseline differences in body mass index were not significant in any meta-regression model, suggesting that this variable is unlikely to be a source of heterogeneity in the meta-analysis results.

In addition to primary and secondary outcomes, maternal biochemical hypoglycaemia was not reported in any study. Additional side effects in the metformin group (mainly gastrointestinal) were reported in five studies, ranging from $2.5 \%$ to $45.7 \% .{ }^{426-29}$ Nevertheless, patients preferred metformin over insulin in the two studies that addressed patient satisfaction. ${ }^{425}$

\section{Metformin versus glibenclamide}

Only two trials compared metformin and glibenclamide head to head, and their characteristics are summarized in supplementary table $2 .{ }^{50}$ Studies were performed in the US and Brazil, and diagnostic criteria were those of Carpenter and Coustan $^{30}$ and the WHO. ${ }^{5}$ Blood glucose cut-off values for initiating drug treatment were lower in one of the trials. ${ }^{5}$ Metformin and glibenclamide groups were similar in baseline characteristics with the exception of higher maternal age (pooled mean difference 1.36 years ( $95 \%$ confidence interval 0.07 to 2.64)) and number of prior pregnancies (pooled mean difference 0.37 pregnancies/patient $(0.20$ to 0.72$)$ ) in the metformin group (see supplementary table 4 ).

Both trials were open label and described the randomization procedure and allocation concealment. Only one of the studies had a published protocol and described sample size calculation. ${ }^{30}$ One study reported one variable less than those described in the methods (macrosomia). ${ }^{5}$ There were no attritions (see supplementary table 3 ), and a sensitivity analysis was not necessary since no study fulfilled criteria for exclusion.

When compared with glibenclamide, metformin was associated with less maternal weight gain (pooled mean difference -2.06 $\mathrm{kg}(-3.98$ to -0.14$)$ ), lower birth weight (pooled mean difference $-209 \mathrm{~g}(-314$ to -104$)$ ), less macrosomia (pooled risk ratio 0.33 (0.13 to 0.81$)$ ), and fewer large for gestational age newborns (pooled risk ratio 0.44 ( 0.21 to 0.92$)$ ). The average treatment failure was $26.8 \%$ (48/179) in the metformin group versus $23.5 \%$ (40/170) in the glibenclamide group. For secondary outcomes, metformin was associated with higher fasting blood glucose during treatment (pooled mean difference $0.15 \mathrm{mmol} / \mathrm{L}(0.00$ to 0.30$)$ ) (table $3 \Downarrow$ ). Funnel plots displayed symmetrical distributions for all the outcomes with the exception of caesarean section. As to outcomes where definition and detection protocol can display more differences, both authors reported data on neonatal hypoglycaemia (similar definition, protocol for detection reported in one study ${ }^{5}$ ) and neonatal intensive care unit stay (not defined in either of the studies).

In the qualitative assessment of birth weight, macrosomia, and fasting blood glucose (outcomes addressed in both studies and with significant differences), the direction, magnitude, and precision of the effects were similar with the exception of a wider interval for macrosomia in the study of Moore et al. ${ }^{30}$ In addition to primary and secondary outcomes, maternal biochemical hypoglycaemia was reported to be similar in both groups in the study of Moore et al. ${ }^{30}$ Additional side effects were not reported.

\section{Discussion}

The results of our present study in women with gestational diabetes requiring drug treatment show that glibenclamide and metformin do not achieve similar short term outcomes when compared with each other or when compared with insulin.

\section{Glibenclamide versus insulin}

The main finding was observed when comparing glibenclamide with insulin. Birth weight was about $100 \mathrm{~g}$ higher, neonatal hypoglycaemia was twofold higher, and macrosomia was more than twofold higher in the glibenclamide group. The magnitude of the difference in these outcomes is relevant for clinical practice. In addition, the higher-although not significant—risk ratios for large for gestational age and severe neonatal hypoglycaemia point in the same direction. It is important to note that neonatal hypoglycaemia and macrosomia are included among highest priority outcomes in a study specifically addressing research needs in gestational diabetes. ${ }^{31}$

Higher birth weight ${ }^{315}{ }^{20}$ and higher rates of macrosomia ${ }^{315}$ and neonatal hypoglycaemia ${ }^{152032}$ with glibenclamide use had already been reported in individual trials ${ }^{320}$ or meta-analyses, ${ }^{15} 32$ but these untoward effects have received little attention to date. For example, the American College of Obstetricians and Gynecologists (ACOG) practice bulletin on gestational diabetes in 2013 indicates that current data show no adverse short term effects from oral diabetic therapy during pregnancy on maternal or neonatal health but that long term outcomes have yet to be studied. ${ }^{9}$ We now report a comprehensive meta-analysis on randomized controlled trials comparing glibenclamide with insulin to provide robust estimators for these outcomes.

The main differences between our present meta-analysis on glibenclamide versus insulin and that by Zeng et $\mathrm{al}^{15}$ are, first, that we included two additional papers ${ }^{2324}$ and, second, that the outcomes addressed in the two meta-analyses were not completely coincident. We did not consider four outcomes included by Zeng and colleagues ${ }^{15}$ (non-severe maternal hypoglycaemia, congenital malformations, hypocalcaemia, polycythaemia), but we considered 17 additional ones (maternal total weight gain, maternal weight gain since entry, maternal severe hypoglycaemia, pregnancy induced hypertension, gestational age at delivery, induction, cord $\mathrm{C}$ peptide and insulin, Apgar score at 1 and 5 minutes, small for gestational age, obstetric trauma, severe neonatal hypoglycaemia, respiratory distress, and mortality (stillbirth, neonatal, and perinatal)).

Our meta-analysis confirms the results of Zeng et al, ${ }^{15}$ but our interpretation is clearly divergent. Although advising some caution, Zeng et al conclude that glibenclamide is a convenient alternative to insulin in developing countries. We consider that the twofold higher neonatal hypoglycaemia and more than twofold higher macrosomia in women treated with glibenclamide are unacceptable; if an alternative to insulin is required, metformin - with a more favourable short term profile-would be a better option. Our third meta-analysis, comparing metformin with glibenclamide, provides confirmation of the untoward effects of glibenclamide.

Even when the few trials addressing cord insulin or $\mathrm{C}$ peptide have not shown any difference, we can only understand the results in the glibenclamide versus insulin comparison in terms of more fetal hyperinsulinism in the glibenclamide arm. As glycaemic control cannot account for this hyperinsulinism, it is 
necessary to consider glibenclamide itself. Reports from Langer et al do not support a significant maternal to fetal transfer of glibenclamide ${ }^{2}$ or its metabolites. ${ }^{33}$

However Hebert et al ${ }^{34}$ describe a maternal to fetal ratio for glibenclamide of 0.7 . They attribute these diverging results to the use of a method with a detection limit of $0.25 \mathrm{ng} / \mathrm{mL},{ }^{34}$ while that of the method of Langer et al was $10 \mathrm{ng} / \mathrm{mL} .{ }^{2}$ We therefore consider that maternal to fetal transfer of glibenclamide is the most likely explanation for the higher birth weight and risk ratios of macrosomia and neonatal hypoglycaemia observed in the glibenclamide group.

\section{Metformin versus insulin}

The meta-analysis of randomized controlled trials comparing metformin with insulin showed differences in seven of the 29 analyzed variables, but the magnitudes were smaller than those for glibenclamide versus insulin. In the metformin group, maternal outcomes were better in terms of total weight gain, weight gain since study entry, postprandial blood glucose, and pregnancy induced hypertension, whereas fetal outcomes were worse in terms of gestational age at delivery and preterm birth and better in terms of severe neonatal hypoglycaemia. Among these variables, weight gain, pregnancy induced hypertension, and neonatal hypoglycaemia are included as highest priority outcomes in a study specifically addressing research needs in gestational diabetes. ${ }^{31}$ Individual trials had previously reported significant differences in the above outcomes (postprandial glycaemia, ${ }^{4}$ gestational age at delivery, ${ }^{4}$ preterm birth, ${ }^{4}$ weight gain, ${ }^{47}$ weight gain since study entry, ${ }^{47}{ }^{29}$ neonatal hypoglycaemia ${ }^{29}$ and severe neonatal hypoglycaemia ${ }^{4}$ ) with the exception of pregnancy induced hypertension, that nevertheless reached significance in a recent meta-analysis. ${ }^{17}$

The main differences between the meta-analysis by Gui et $\mathrm{al}^{17}$ and the present study are, first, that we included an additional randomized controlled trial ${ }^{29}$ and, second, that the outcomes addressed in the two meta-analyses were not completely coincident. We did not consider three outcomes included by Gui et al (congenital malformations, cord $\mathrm{pH}$, and phototherapy) ${ }^{17}$ but we considered nine additional ones (maternal total weight gain and severe hypoglycaemia, induction, cord $\mathrm{C}$ peptide and insulin, Apgar score at 1 minute, macrosomia, severe neonatal hypoglycaemia, and mortality (stillbirth, neonatal, perinatal)). Our meta-analysis confirms the results of Gui et $\mathrm{al}^{17}$ and adds a lower total maternal weight gain and a lower occurrence of severe neonatal hypoglycaemia in the metformin group.

It is important to note that, although metformin and insulin groups in the current study were similar in most baseline characteristics, prepregnancy body mass index was higher by 0.78 in the metformin group. It is likely that this higher body mass index influenced outcomes such as pregnancy induced hypertension, ${ }^{35}$ pre-eclampsia, ${ }^{35}$ birth weight,${ }^{35}$ large for gestational age, ${ }^{35}$ and neonatal hypoglycaemia. ${ }^{36}$ If treatment groups had had a similar baseline body mass index, differences in outcomes could have been more apparent (pregnancy induced hypertension, severe neonatal hypoglycaemia) or become significant (pre-eclampsia, birth weight, large for gestational age, any neonatal hypoglycaemia).

Regarding potential mechanisms mediating differences with metformin use, the lower postprandial blood glucose observed with metformin $(-0.14 \mathrm{mmol} / \mathrm{L})$ seems insufficient to account for the lower rate in pregnancy induced hypertension and severe neonatal hypoglycaemia. ${ }^{37}$ The effect of metformin on maternal weight gain could be expected considering the effect of metformin outside pregnancy, while that on pregnancy induced hypertension could be attributed to improvements in insulin resistance, inflammation, or endothelial function. The effect of metformin on gestational age-small, but sufficient to increase the rate of preterm birth by $50 \%$ - is inconsistent with the slightly better postprandial blood glucose and the reduced rate of pregnancy induced hypertension. This effect of metformin on gestational age could be attributed to metformin itself. However, it is of note that in one report in women with polycystic ovary syndrome, metformin treatment was associated with a lower rate of preterm birth, ${ }^{38}$ and the authors indicated that underlying mechanism(s) could only be speculated on. It should also be pointed out that in our study, and contrary to what might be expected, a lower gestational age at birth in the metformin group was associated with less neonatal hypoglycaemia. Again, an independent effect of metformin could be hypothesized.

Despite the fact that side effects were more common in women treated with metformin, it was the favoured drug in the studies addressing preference.

\section{Metformin versus glibenclamide}

The meta-analysis of the two randomized controlled trials comparing metformin with glibenclamide showed differences in five of the 30 analyzed variables. Differences in total maternal weight gain, birth weight, macrosomia, and large for gestational age were roughly the sum of the effects seen in the first two meta-analyses. However, the results in three outcomes were unexpected after the findings of the first two comparisons: in the metformin group, we would expect a lower rate of neonatal hypoglycaemia, no difference for fasting blood glucose, and a higher rate of treatment failure. We have no explanation for the difference in fasting blood glucose, while the lack of significance for neonatal hypoglycaemia and treatment failure could be due to the relatively small number of included subjects. The higher fasting blood glucose in the metformin group $(0.15 \mathrm{mmol} / \mathrm{L})$ cannot account for the differences in clinical outcomes, first, because it cannot explain the lower maternal weight gain and, second, because it would have increased outcomes related to birth weight rather than decrease them. Overall, we consider the results of this comparison as confirmatory of the maternal weight gain and outcomes related to birth weight reported in the first two. The results of the qualitative assessment support the results of the meta-analysis for outcomes with significant differences.

Among the variables where significant differences were reached, maternal weight gain, macrosomia, and large for gestational age are included as highest priority outcomes in a study specifically addressing research needs in gestational diabetes. ${ }^{31}$ In the three meta-analyses, and for most outcomes, definition and active ascertainment were not an issue. For outcomes where this could be important, definitions and detection protocol when provided were reasonably similar with the exception of neonatal hypoglycaemia. Nevertheless, heterogeneity was very low for most of these variables.

The main limitation of this study is that we have performed meta-analyses of aggregated patient data, whereas using individual patient data would have allowed better adjustment for baseline characteristics. Its main strength is to be the most comprehensive systematic review and meta-analysis addressing glibenclamide and metformin versus insulin or versus each other in the treatment of women with gestational diabetes requiring drug treatment. 


\section{Conclusion}

Oral agents are clearly more attractive than insulin to treat women with gestational diabetes, but attainment of similar or better results should be a prerequisite for their use. The results of the three meta-analyses presented here indicate that, in the short term at least, this is not the case for glibenclamide (higher birth weight and more macrosomia and neonatal hypoglycaemia). For metformin, results were better for maternal outcomes in terms of weight gain and pregnancy induced hypertension but uneven for fetal outcomes: more preterm birth and less severe neonatal hypoglycaemia. It is important to bear in mind, however, that metformin is associated with a higher rate of treatment failure and that its long term safety remains unknown.

As to future research, we consider that: (1) meta-analyses of individual patient data would allow adjustment for differences in baseline characteristics, especially important in the comparison of metformin with insulin; (2) although a definite proof of glibenclamide producing more fetal hyperinsulinism is lacking, current evidence discourages further trials using this drug; (3) studies addressing mechanisms underlying earlier delivery and less neonatal hypoglycaemia in women treated with metformin would be of interest.

We conclude that, in the short term, in women with gestational diabetes requiring drug treatment, glibenclamide is clearly inferior to both insulin and metformin while metformin (plus insulin when required) performs slightly better than insulin. According to these results, glibenclamide should not be used for the treatment of women with gestational diabetes if metformin or insulin are available.

We thank Carolyn Newey, author editor that has reviewed the draft written by the authors. We also thank the authors of randomized controlled trials who provided the requested information.

Preliminary results of this study have been presented at the 45th Meeting of the Diabetic Pregnancy Study Group held in Malta in 2013 and at the XXV meeting of the Sociedad Española de Diabetes held in Pamplona in 2014; corresponding abstracts were published in the 45th DPSG abstract book and in Av Diabetol 2014;30(Espec Congr):209.

Contributors: $\mathrm{RC}$ is the guarantor of this article. She had full access to all of the data in the study and takes responsibility for the integrity of the data and the accuracy of the data analysis. MB, AGP and RC conceived and designed the study, MB and AGP performed data acquisition. MB, AGP, IS, MR, IG and RC analysed and interpreted the data and carried out the statistical analyses. MB, AGP and RC drafted the manuscript. All authors critically revised the manuscript for important intellectual content.

Funding: None. The study sponsor is Fundació Institut de Recerca de l'Hospital de la Santa Creu i Sant Pau; the sponsor was not involved in the design or execution of the systematic review or in the writing of the manuscript.

Competing interests: All authors have completed the ICMJE uniform disclosure form at www.icmje.org/coi_disclosure.pdf and declare: declare no support from any organisation for the submitted work; no financial relationships with any organisations that might have an interest in the submitted work in the previous three years, though MB, AGP, and RC have received travel grants from Lilly, NovoNordisk, and Sanofi Aventis; and no other relationships or activities that could appear to have influenced the submitted work.

Ethical approval: Not required

1 Hunt KJ, Schuller KL. The increasing prevalence of diabetes in pregnancy. Obstet Gynecol Clin North Am 2007;34:173-99.

2 Langer O, Conway DL, Berkus MD, Xenakis EM, Gonzales O. A comparison of glyburide and insulin in women with gestational diabetes mellitus. N Engl J Med 2000;343:1134-8.
3 Lain KY, Garabedian MJ, Daftary A, Jeyabalan A. Neonatal adiposity following maternal treatment of gestational diabetes with glyburide compared with insulin. Am J Obstet Gynecol 2009;200:501.e501-506.

4 Rowan JA, Hague WM, Gao W, Battin MR, Moore MP. Metformin versus insulin for the treatment of gestational diabetes. N Engl J Med 2008;358:2003-15.

5 Silva JC, Fachin DR, Coral ML, Bertini AM. Perinatal impact of the use of metformin and glyburide for the treatment of gestational diabetes mellitus. J Perinat Med 2012;40:225-8.

6 Jacobson GF, Ramos GA, Ching JY, Kirby RS, Ferrara A, Field DR. Comparison of glyburide and insulin for the management of gestational diabetes in a large managed care organization. Am J Obstet Gynecol 2005;193:118-24.

7 Metzger BE, Buchanan TA, Coustan DR, de Leiva A, Dunger DB, Hadden DR, et al. Summary and recommendations of the Fifth International Workshop-Conference on Gestational Diabetes Mellitus. Diabetes Care 2007;30(suppl 2):S251-60.

8 National Institute for Health and Care Excellence. Diabetes in pregnancy: management of diabetes and its complications from pre-conception to the postnatal period. (Clinical guideline 63.) 2008. www.nice.org.uk/guidance/cg63/.

9 Committee on Practice Bulletins-Obstetrics. Practice bulletin No. 137: gestational diabetes mellitus. Obstet Gynecol 2013;122:406-16.

10 Coetzee EJ. Pregnancy and diabetes scenario around the world: Africa. Int J Gynaecol Obstet 2009;104:S39-41.

11 Goh JE, Sadler L, Rowan J. Metformin for gestational diabetes in routine clinical practice. Diabetic Med 2011;28:1082-7.

12 Camelo Castillo W, Boggess K, Sturmer T, Brookhart MA, Benjamin DK Jr, Jonsson Funk M. Trends in glyburide compared with insulin use for gestational diabetes treatment in the United States, 2000-2011. Obstet Gynecol 2014;123:1177-84.

13 Heilmaier C, Thielscher C, Ziller M, Altmann V, Kostev K. Use of antidiabetic agents in the treatment of gestational diabetes mellitus in Germany, 2008-2012. J Obstet Gynaecol Res 2014;40:1592-7.

14 Moretti ME, Rezvani M, Koren G. Safety of glyburide for gestational diabetes: a meta-analysis of pregnancy outcomes. Ann Pharmacother 2008;42:483-90.

15 Zeng YC, Li MJ, Chen Y, Jiang L, Wang SM, Mo XL, et al. The use of glyburide in the management of gestational diabetes mellitus: a meta-analysis. Adv Med Sci 2014:59:95-101.

16 Dhulkotia JS, Ola B, Fraser R, Farrell T. Oral hypoglycemic agents vs insulin in management of gestational diabetes: a systematic review and metaanalysis. Am J Obstet Gynecol 2010;203:457.e1-9.

17 Gui J, Liu Q, Feng L. Metformin vs insulin in the management of gestational diabetes: a meta-analysis. PloS One 2013;8:e64585.

18 Su DF, Wang XY. Metformin vs insulin in the management of gestational diabetes: a systematic review and meta-analysis. Diabetes Res Clin Pract 2014;104:353-7.

19 Higgins JPT, Altman DG, Sterne JAC, eds. Chapter 8: Assessing risk of bias in included studies. In: Higgins JPT, Green S, eds. Cochrane Handbook for Systematic Reviews of Interventions Version 5.1.0 . Cochrane Collaboration, 2011. www.cochrane-handbook. org.

20 Silva JC, Bertini AM, Taborda W, Becker F, Bebber FR, Aquim GM, et al. [Glibenclamide in the treatment for gestational diabetes mellitus in a compared study to insulin]. Arq Bras Endocrinol Metabol 2007;51:541-6.

21 Anjalakshi C, Balaji V, Balaji MS, Seshiah V. A prospective study comparing insulin and glibenclamide in gestational diabetes mellitus in Asian Indian women. Diabetes Res Clin Pract 2007;76:474-5.

22 Ogunyemi D, Jesse M, Davidson M. Comparison of glyburide versus insulin in management of gestational diabetes mellitus. Endocr Pract 2007;13:427-8.

23 Mukhopadhyay P, Bag TS, Kyal A, Saha DP, Khalid N. Oral hypoglycemic glibenclamide: can it be a substitute to insulin in the management of gestational diabetes mellitus? A comparative study. J SAFOG 2012;4:28-31.

24 Tempe A, Mayanglambam RD. Glyburide as treatment option for gestational diabetes mellitus. J Obstet Gynaecol Res 2013:39:1147-52.

25 Moore LE, Briery CM, Clokey D, Martin RW, Williford NJ, Bofill JA et al. Metformin and insulin in the management of gestational diabetes mellitus: preliminary results of a comparison. J Reprod Med 2007;52:1011-5.

26 ljas H, Vaarasmaki M, Morin-Papunen L, Keravuo R, Ebeling T, Saarela T, et al. Metformin should be considered in the treatment of gestational diabetes: a prospective randomised study. BJOG 2011;118:880-5.

27 Niromanesh S, Alavi A, Sharbaf FR, Amjadi N, Moosavi S, Akbari S. Metformin compared with insulin in the management of gestational diabetes mellitus: a randomized clinical trial. Diabetes Res Clin Pract 2012;98:422-9.

28 Tertti K, Ekblad U, Koskinen P, Vahlberg T, Ronnemaa T. Metformin vs. insulin in gestational diabetes. A randomized study characterizing metformin patients needing additional insulin. Diabetes Obes Metab 2013;15:246-51.

29 Spaulonci CP, Bernardes LS, Trindade TC, Zugaib M, Francisco RP. Randomized trial of metformin vs insulin in the management of gestational diabetes. Am J Obstet Gynecol 2013;209:34.e1-7.

30 Moore LE, Clokey D, Rappaport VJ, Curet LB. Metformin compared with glyburide in gestational diabetes: a randomized controlled trial. Obstet Gynecol 2010;115:55-9.

31 Bennett WL, Robinson KA, Saldanha IJ, Wilson LM, Nicholson WK. High priority research needs for gestational diabetes mellitus. J Womens Health 2012;21:925-32.

32 Waugh N, Royle P, Clar C, Henderson R, Cummins E, Hadden D, et al. Screening for hyperglycaemia in pregnancy: a rapid update for the National Screening Committee. Health Technol Assess 2010;14:1-183.

33 Elliott $\mathrm{BD}$, Langer $\mathrm{O}$, Schenker $\mathrm{S}$, Johnson RF. Insignificant transfer of glyburide occurs across the human placenta. Am J Obstet Gynecol 1991;165:807-12.

34 Hebert MF, Ma X, Naraharisetti SB, Krudys KM, Umans JG, Hankins GD, et al. Are we optimizing gestational diabetes treatment with glyburide? The pharmacologic basis for better clinical practice. Clin Pharmacol Ther 2009;85:607-14.

35 Siega-Riz AM, Siega-Riz AM, Laraia B. The implications of maternal overweight and obesity on the course of pregnancy and birth outcomes. Maternal Child Health J 2006;10(5 suppl):S153-6.

36 Garcia-Patterson A, Aulinas A, Maria MA, Ubeda J, Orellana I, Ginovart G, et al. Maternal body mass index is a predictor of neonatal hypoglycemia in gestational diabetes mellitus. $J$ Clin Endocrinol Metab 2012;97:1623-8.

37 International Association of Diabetes and Pregnancy Study Groups Consensus Pane Metzger BE, Gabbe SG, Persson B, Buchanan TA, Catalano PA, et al. International association of diabetes and pregnancy study groups recommendations on the diagnosis and classification of hyperglycemia in pregnancy. Diabetes Care 2010;33:676-82. 


\section{What is already known on this topic}

Oral agents are increasingly used to treat gestational diabetes, and current guidelines consider their use even though information on their safety is limited

Although several meta-analyses of randomized controlled trials addressing oral agents have been published, a comprehensive study including glibenclamide versus insulin, metformin versus insulin, and metformin versus glibenclamide has not been performed

\section{What this study adds}

In the short term, in women with gestational diabetes requiring drug treatment, glibenclamide is clearly inferior to both insulin and metformin whereas metformin (plus insulin when required) performs slightly better than insulin

38 Vanky E, De Zegher F, Diaz M, Ibanez L, Carlsen SM. On the potential of metformin to prevent preterm delivery in women with polycystic ovary syndrome - an epi-analysis. Acta Obstet Gynecol Scand 2012;91:1460-4.

Accepted: 25 November 2014
Cite this as: BMJ 2015;350:h102

This is an Open Access article distributed in accordance with the Creative Commons Attribution Non Commercial (CC BY-NC 4.0) license, which permits others to distribute, remix, adapt, build upon this work non-commercially, and license their derivative works on different terms, provided the original work is properly cited and the use is non-commercial. See: http://creativecommons.org/licenses/by-nc/4.0/. 


\section{Tables}

Table 1| Meta-analysis of randomized controlled trials comparing outcomes from treatment with glibenclamide $\mathbf{v}$ insulin in women with gestational diabetes

\begin{tabular}{|c|c|c|c|c|c|c|c|}
\hline \multirow[b]{2}{*}{ Outcomes } & \multirow[b]{2}{*}{$\begin{array}{l}\text { No of } \\
\text { studies }\end{array}$} & \multicolumn{2}{|c|}{ No of patients treated } & \multirow[b]{2}{*}{$\begin{array}{l}\text { Mean difference } \\
(95 \% \mathrm{Cl})\end{array}$} & \multirow[b]{2}{*}{ Relative risk (95\% Cl) } & \multirow[b]{2}{*}{$P$ value } & \multirow[b]{2}{*}{$I^{2}$ value } \\
\hline & & $\begin{array}{c}\text { With } \\
\text { glibenclamide }\end{array}$ & With insulin & & & & \\
\hline \multicolumn{8}{|l|}{ Primary outcomes } \\
\hline $\mathrm{HbA}_{1 \mathrm{c}}$ level at end of third trimester (\%) & 3 & 241 & 246 & $-0.03(-0.25 \text { to } 0.18)^{*}$ & - & 0.75 & 52 \\
\hline Severe maternal hypoglycaemia (\%) & 4 & 277 & 278 & - & $0 \vee 0$ & - & - \\
\hline Pre-eclampsia (\%) & 4 & 275 & 284 & - & $0.90(0.51$ to 1.61$)$ & 0.73 & 0 \\
\hline Maternal weight gain $(\mathrm{kg})$ & 2 & 233 & 239 & $-0.5(-1.6$ to 0.62$)$ & - & 0.38 & 21 \\
\hline Caesarean section (\%) & 4 & 286 & 297 & - & $0.89(0.71$ to 1.11$)$ & 0.30 & 3 \\
\hline Gestational age at delivery (weeks) & 6 & 384 & 391 & $0.05(-0.18$ to 0.28$)$ & - & 0.69 & 14 \\
\hline Preterm birth (\%) & 5 & 316 & 325 & - & $0.87(0.57$ to 1.33$)$ & 0.52 & 0 \\
\hline Birth weight $(\mathrm{g})$ & 7 & 394 & 404 & 109 (35.9 to 181$)$ & - & 0.003 & 0 \\
\hline Macrosomia (\%) & 6 & 346 & 355 & - & 2.62 (1.35 to 5.08$)$ & 0.004 & 34 \\
\hline Large for gestational age (\%) & 5 & 314 & 323 & - & $1.87(0.76 \text { to } 4.61)^{\star}$ & 0.17 & 52 \\
\hline Small for gestational age (\%) & 4 & 284 & 293 & - & $1.01(0.56$ to 1.82$)$ & 0.97 & 9 \\
\hline Any neonatal hypoglycaemia (\%) & 7 & 386 & 400 & - & 2.04 (1.30 to 3.20$)$ & 0.002 & 0 \\
\hline Perinatal mortality (\%) & 3 & 277 & 287 & - & $1.45(0.29$ to 7.21$)$ & 0.65 & 0 \\
\hline \multicolumn{8}{|l|}{ Secondary outcomes } \\
\hline Fasting blood glucose (mmol/L) & 3 & 274 & 280 & $0.06(-0.06$ to 0.19$)$ & - & 0.33 & 0 \\
\hline Postprandial blood glucose (mmol/L) & 4 & 284 & 293 & $0.08(-0.08$ to 0.24$)$ & - & 0.34 & 0 \\
\hline Maternal weight gain since entry (kg) & 0 & - & - & - & - & - & - \\
\hline Pregnancy induced hypertension (\%) & 0 & - & - & - & - & - & - \\
\hline Induction (\%) & 0 & - & - & - & - & - & - \\
\hline Cord C peptide (nmol/L) & 1 & 31 & 28 & $0.07(-0.14$ to 0.27$)$ & - & 0.63 & - \\
\hline Cord insulin (pmol/L) & 3 & 242 & 244 & $-4.31(-20.4$ to 11.7$)$ & - & 0.60 & 0 \\
\hline 1 minute Apgar score < $7(\%)$ & 0 & - & - & - & - & - & - \\
\hline 5 minute Apgar score <7 (\%) & 0 & - & - & - & - & - & - \\
\hline Obstetric trauma (\%) & 2 & 73 & 77 & - & $0 \vee 0$ & - & - \\
\hline Severe neonatal hypoglycaemia† (\%) & 3 & 81 & 90 & - & $5.31(0.63$ to 44.9$)$ & 0.13 & 0 \\
\hline Neonatal jaundice (\%) & 4 & 271 & 278 & - & $1.44(0.68$ to 3.06$)$ & 0.34 & 0 \\
\hline $\begin{array}{l}\text { Neonatal respiratory distress syndrome } \\
(\%)\end{array}$ & 2 & 231 & 233 & - & 0.61 (0.19 to 1.92$)$ & 0.39 & 0 \\
\hline Stillbirth (\%) & 5 & 317 & 326 & - & $1.60(0.46$ to 5.49$)$ & 0.46 & 0 \\
\hline Neonatal mortality (\%) & 2 & 244 & 251 & - & $1.01(0.06$ to 16.0$)$ & 0.99 & - \\
\hline NICU admission (\%) & 4 & 302 & 306 & - & $0.97(0.56$ to 1.69$)$ & 0.92 & 0 \\
\hline
\end{tabular}

$\mathrm{I}^{2}=$ Heterogeneity. $\mathrm{HbA}_{10}=$ glycated haemoglobin. $\mathrm{NICU}=$ neonatal intensive care unit.

${ }^{*}$ Estimated using a random effects model because $\mathrm{I}^{2}>50 \%$

†Defined by authors or requiring intravenous glucose or NICU admittance. 
Table 2| Meta-analysis of randomized controlled trials comparing outcomes from treatment with metformin $\mathbf{v}$ insulin in women with gestational diabetes

\begin{tabular}{|c|c|c|c|c|c|c|c|}
\hline \multirow[b]{2}{*}{ Outcomes } & \multirow{2}{*}{$\begin{array}{l}\text { No of } \\
\text { studies }\end{array}$} & \multicolumn{2}{|c|}{ No of patients treated } & \multirow{2}{*}{$\begin{array}{l}\text { Mean difference } \\
\quad(95 \% \mathrm{Cl})\end{array}$} & \multirow[b]{2}{*}{ Relative risk $(95 \% \mathrm{Cl})$} & \multirow[b]{2}{*}{$P$ value } & \multirow[b]{2}{*}{$I^{2}$ value } \\
\hline & & With metformin & With insulin & & & & \\
\hline \multicolumn{8}{|l|}{ Primary outcomes } \\
\hline $\mathrm{HbA}_{1 \mathrm{c}}$ level at end of third trimester (\%) & 4 & 508 & 533 & $-0.04(-0.09$ to 0.02$)$ & - & 0.21 & 24 \\
\hline Severe maternal hypoglycaemia (\%) & 4 & 268 & 268 & - & $0 v 0$ & - & - \\
\hline Pre-eclampsia (\%) & 5 & 646 & 653 & - & $0.82(0.56$ to 1.20$)$ & 0.32 & 0 \\
\hline Maternal weight gain $(\mathrm{kg})$ & 4 & 468 & 505 & $-1.14(-2.22 \text { to }-0.06)^{\star}$ & - & 0.04 & 64 \\
\hline Caesarean section (\%) & 6 & 678 & 684 & & $0.97(0.85$ to 1.12$)$ & 0.71 & 17 \\
\hline Gestational age at delivery (weeks) & 6 & 678 & 684 & $-0.16(-0.30$ to -0.02$)$ & - & 0.03 & 0 \\
\hline Preterm birth (\%) & 5 & 646 & 653 & - & $1.50(1.04$ to 2.16$)$ & 0.03 & 0 \\
\hline Birth weight (g) & 6 & 678 & 684 & $-31.4(-86.5$ to 23.6$)$ & - & 0.26 & 4 \\
\hline Macrosomia (\%) & 6 & 678 & 684 & - & $0.96(0.73$ to 1.27$)$ & 0.80 & 13 \\
\hline Large for gestational age (\%) & 5 & 646 & 653 & - & 0.88 (0.69 to 1.11$)$ & 0.28 & 22 \\
\hline Small for gestational age (\%) & 5 & 646 & 653 & - & 0.88 (0.58 to 1.33$)$ & 0.53 & 0 \\
\hline Any neonatal hypoglycaemia (\%) & 6 & 676 & 684 & - & $0.78(0.60$ to 1.01$)$ & 0.06 & 0 \\
\hline Perinatal mortality $(\%)$ & 4 & 600 & 607 & - & $1.0(0.14$ to 7.12$)$ & 0.99 & 0 \\
\hline \multicolumn{8}{|l|}{ Secondary outcomes } \\
\hline Fasting blood glucose (mmol/L) & 4 & 521 & 527 & $0.04(-0.03$ to 0.11$)$ & - & 0.24 & 49 \\
\hline Postprandial blood glucose (mmol/L) & 4 & 521 & 527 & $-0.14(-0.22$ to -0.05$)$ & - & 0.002 & 38 \\
\hline Maternal weight gain since entry $(\mathrm{kg})$ & 4 & 565 & 533 & $-1.23(-1.72 \text { to }-0.73)^{*}$ & - & $<0.001$ & 61 \\
\hline Pregnancy induced hypertension (\%) & 3 & 553 & 557 & - & $0.53(0.31$ to 0.90$)$ & 0.02 & 0 \\
\hline Induction (\%) & 3 & 520 & 527 & - & 0.91 (0.81 to 1.02$)$ & 0.11 & 46 \\
\hline Cord C peptide (nmol/L) & 0 & - & - & - & - & - & - \\
\hline Cord insulin (pmol/L) & 1 & 220 & 234 & $7.64(-92.7$ to 108$)$ & - & 0.88 & - \\
\hline 1 minute Apgar score < $7(\%)$ & 0 & - & - & - & - & - & - \\
\hline 5 minute Apgar score <7 (\%) & 1 & 363 & 370 & - & $3.06(0.32$ to 29.3$)$ & 0.33 & - \\
\hline Obstetric trauma (\%) & 2 & 472 & 477 & - & 0.86 (0.45 to 1.63$)$ & 0.64 & 2 \\
\hline Severe neonatal hypoglycaemia† (\%) & 3 & 519 & 527 & - & $0.62(0.42$ to 0.94$)$ & 0.02 & 48 \\
\hline Neonatal jaundice (\%) & 6 & 677 & 684 & - & $0.90(0.67$ to 1.21$)$ & 0.47 & 0 \\
\hline Neonatal respiratory distress syndrome (\%) & 5 & 630 & 634 & - & $1.30(0.70$ to 2.41$)$ & 0.40 & 0 \\
\hline Stillbirth (\%) & 5 & 632 & 638 & - & $1.38(0.27$ to 7.02$)$ & 0.69 & 0 \\
\hline Neonatal mortality (\%) & 4 & 599 & 606 & - & $0 v 0$ & - & - \\
\hline NICU admission (\%) & 5 & 631 & 638 & - & $0.87(0.70$ to 1.09$)$ & 0.23 & 0 \\
\hline
\end{tabular}

$\mathrm{I}^{2}=$ Heterogeneity. $\mathrm{HbA}_{10}=$ glycated haemoglobin. $\mathrm{NICU}=$ neonatal intensive care unit.

${ }^{*}$ Estimated using a random effects model because $\mathrm{I}^{2}>50 \%$

†Defined by authors or requiring intravenous glucose or NICU admittance. 
Table 3| Meta-analysis of randomized controlled trials comparing outcomes from treatment with metformin $\mathbf{v}$ glibenclamide in women with gestational diabetes

\begin{tabular}{|c|c|c|c|c|c|c|c|}
\hline \multirow[b]{2}{*}{ Outcomes } & \multirow[b]{2}{*}{$\begin{array}{c}\text { No of } \\
\text { studies }\end{array}$} & \multicolumn{2}{|c|}{ No of patients treated } & \multirow[b]{2}{*}{$\begin{array}{l}\text { Mean difference } \\
(95 \% \mathrm{Cl})\end{array}$} & \multirow[b]{2}{*}{ Relative risk (95\% Cl) } & \multirow[b]{2}{*}{$P$ value } & \multirow[b]{2}{*}{$I^{2}$ value } \\
\hline & & With metformin & $\begin{array}{c}\text { With } \\
\text { glibenclamide }\end{array}$ & & & & \\
\hline \multicolumn{8}{|l|}{ Primary outcomes } \\
\hline $\mathrm{HbA}_{1 \mathrm{c}}$ level at end of third trimester (\%) & 1 & 104 & 96 & $-0.11(-0.34$ to 0.12$)$ & - & 0.34 & - \\
\hline Severe maternal hypoglycaemia (\%) & 1 & 104 & 96 & - & $0 \vee 0$ & - & - \\
\hline Pre-eclampsia (\%) & 2 & 179 & 170 & - & 0.95 (0.28 to 3.25$)$ & 0.94 & 0 \\
\hline Maternal weight gain $(\mathrm{kg})$ & 1 & 104 & 96 & $\begin{array}{c}-2.06(-3.98 \text { to } \\
-0.14)\end{array}$ & - & 0.04 & - \\
\hline Caesarean section (\%) & 2 & 179 & 170 & - & $1.99(0.32 \text { to } 12.4)^{*}$ & 0.46 & 84 \\
\hline Treatment failure (\%) & 2 & 179 & 170 & - & $1.23(0.42 \text { to } 3.54)^{\star}$ & 0.71 & 87 \\
\hline Gestational age at delivery (weeks) & 2 & 179 & 170 & $-0.11(-0.40$ to 0.19$)$ & - & 0.47 & 0 \\
\hline Preterm birth (\%) & 1 & 104 & 96 & - & $1.15(0.32$ to 4.17$)$ & 0.83 & - \\
\hline Birth weight (g) & 2 & 179 & 170 & $-209(-314$ to -104$)$ & - & $<0.001$ & 0 \\
\hline Macrosomia (\%) & 2 & 179 & 170 & - & 0.33 (0.13 to 0.81$)$ & 0.02 & 0 \\
\hline Large for gestational age (\%) & 1 & 104 & 96 & - & 0.44 (0.21 to 0.92$)$ & 0.03 & - \\
\hline Small for gestational age (\%) & 1 & 104 & 96 & - & 1.23 (0.28 to 5.36$)$ & 0.78 & - \\
\hline Any neonatal hypoglycaemia (\%) & 2 & 178 & 169 & - & $0.86(0.42$ to 1.77$)$ & 0.68 & 0 \\
\hline Perinatal mortality (\%) & 1 & 104 & 96 & - & $0.92(0.06$ to 14.6$)$ & 0.95 & - \\
\hline \multicolumn{8}{|l|}{ Secondary outcomes } \\
\hline Fasting blood glucose (mmol/L) & 2 & 179 & 170 & $0.15(0.00$ to 0.30$)$ & - & 0.04 & 0 \\
\hline Postprandial blood glucose (mmol/L) & 2 & 179 & 170 & $-0.04(-0.27$ to 0.19$)$ & - & 0.74 & 0 \\
\hline Maternal weight gain since entry (kg) & 0 & - & - & - & - & - & - \\
\hline Pregnancy induced hypertension (\%) & 0 & - & - & - & - & - & - \\
\hline Induction (\%) & 0 & - & - & - & - & - & - \\
\hline Cord C peptide (nmol/L) & 0 & - & - & - & - & - & - \\
\hline Cord insulin (pmol/L) & 0 & - & - & - & - & - & - \\
\hline 1 minute Apgar score < $7(\%)$ & 1 & 103 & 95 & - & $0.46(0.04$ to 5.00$)$ & 0.52 & - \\
\hline 5 minute Apgar score <7 (\%) & 2 & 178 & 169 & - & 2.77 (0.10 to 67.2$)$ & 0.53 & - \\
\hline Obstetric trauma (\%) & 0 & - & - & - & - & - & - \\
\hline Severe neonatal hypoglycaemia† (\%) & 0 & - & - & - & - & - & - \\
\hline Neonatal jaundice (\%) & 0 & - & - & - & - & - & - \\
\hline $\begin{array}{l}\text { Neonatal respiratory distress syndrome } \\
(\%)\end{array}$ & 0 & - & - & - & - & - & - \\
\hline Stillbirth (\%) & 1 & 104 & 96 & - & $0.92(0.06$ to 14.6$)$ & 0.95 & - \\
\hline Neonatal mortality (\%) & 1 & 103 & 95 & - & $0 \vee 0$ & - & - \\
\hline NICU admission (\%) & 2 & 178 & 169 & - & 1.52 (0.65 to 3.55$)$ & 0.33 & 1 \\
\hline
\end{tabular}

$\mathrm{I}^{2}=$ Heterogeneity. $\mathrm{HbA}_{1 \mathrm{c}}=$ glycated haemoglobin. $\mathrm{NICU}=$ neonatal intensive care unit.

${ }^{*}$ Estimated using a random effects model because $I^{2}>50 \%$

†Defined by authors or requiring intravenous glucose or NICU admittance. 


\section{Figures}

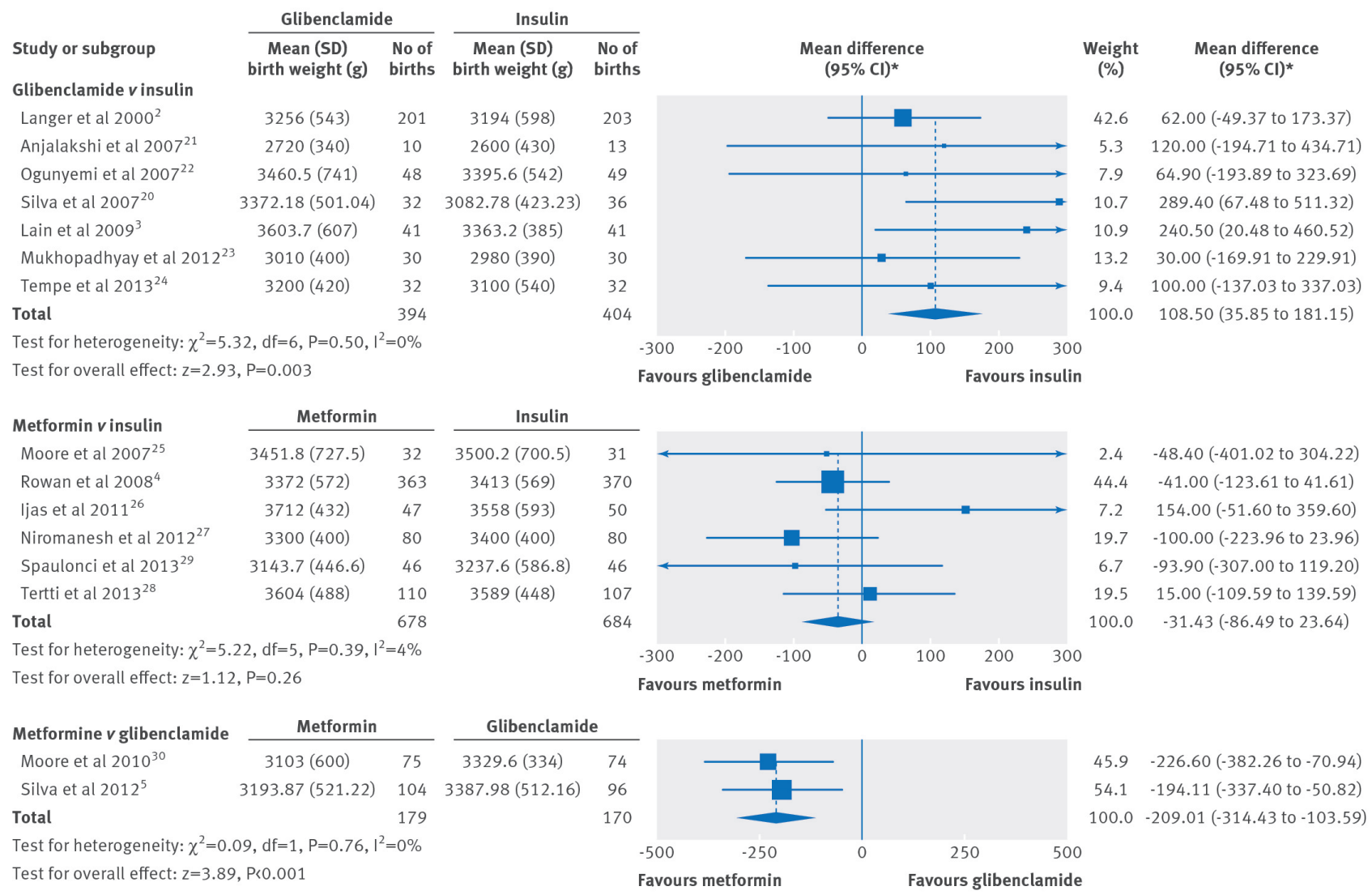

*Instrumental variable fixed effects model

Fig 1 Forest plots of birth weight in the meta-analyses comparing glibenclamide and metformin with insulin or with each other in women with gestational diabetes 


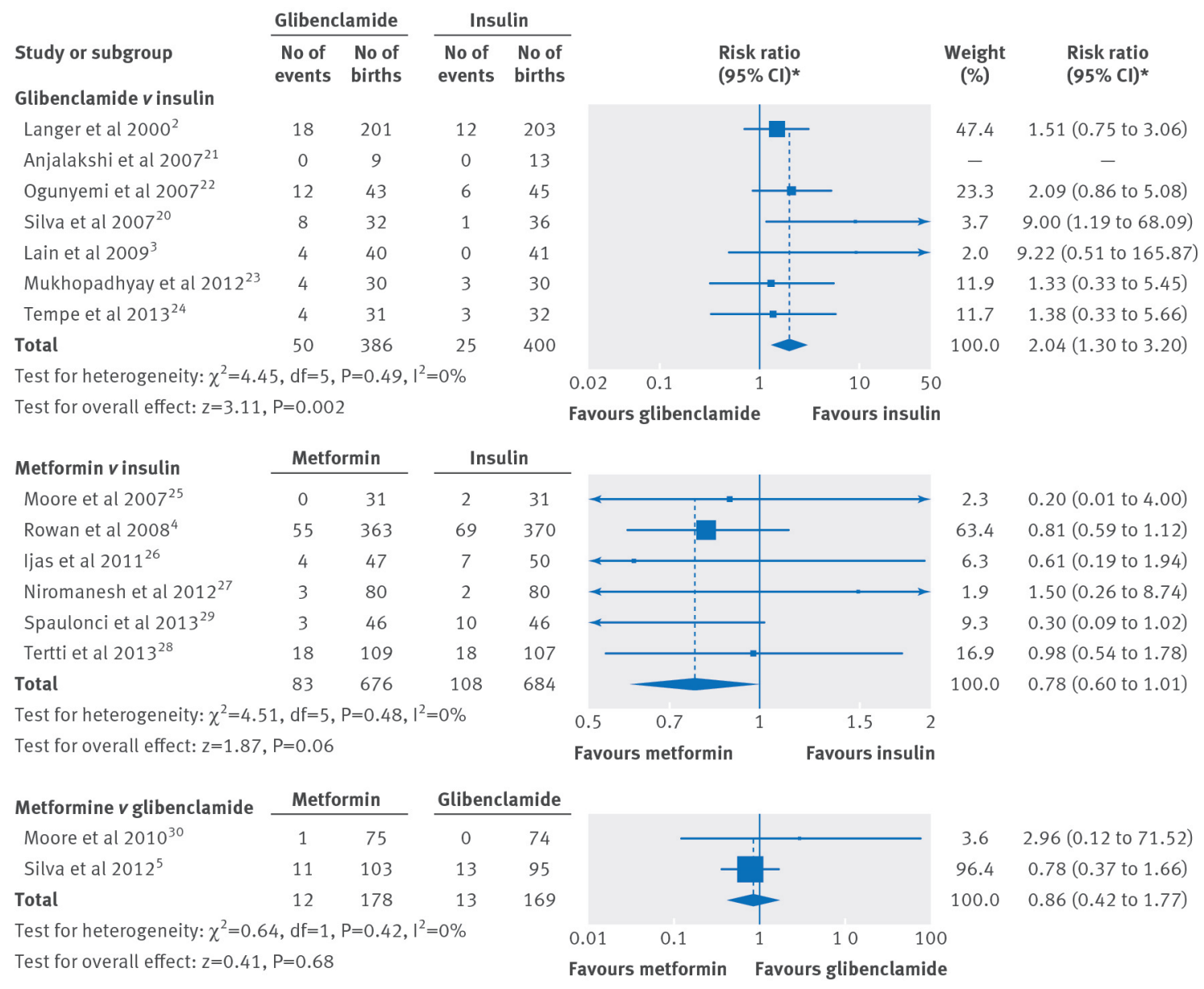

*Mantel-Haenszel fixed effects model

Fig 2 Forest plots of any neonatal hypoglycaemia in the meta-analyses comparing glibenclamide and metformin with insulin or with each other in women with gestational diabetes 